\title{
Predictability of Top of Descent Location for Operational Idle-thrust Descents
}

\author{
Laurel L. Stell* \\ NASA Ames Research Center, Moffett Field, CA 94035
}

\begin{abstract}
To enable arriving aircraft to fly optimized descents computed by the flight management system (FMS) in congested airspace, ground automation must accurately predict descent trajectories. To support development of the trajectory predictor and its uncertainty models, commercial flights executed idle-thrust descents at a specified descent speed, and the recorded data included the specified descent speed profile, aircraft weight, and the winds entered into the FMS as well as the radar data. The FMS computed the intended descent path assuming idle thrust after top of descent (TOD), and the controllers and pilots then endeavored to allow the FMS to fly the descent to the meter fix with minimal human intervention. The horizontal flight path, cruise and meter fix altitudes, and actual TOD location were extracted from the radar data. Using approximately 70 descents each in Boeing 757 and Airbus 319/320 aircraft, multiple regression estimated TOD location as a linear function of the available predictive factors. The cruise and meter fix altitudes, descent speed, and wind clearly improve goodness of fit. The aircraft weight improves fit for the Airbus descents but not for the B757. Except for a few statistical outliers, the residuals have absolute value less than $5 \mathrm{nmi}$. Thus, these predictive factors adequately explain the TOD location, which indicates the data do not include excessive noise.
\end{abstract}

\section{Nomenclature}

b Least squares estimate of $\boldsymbol{\beta}$

$I_{\mathrm{W}, \mathrm{FMS}}$ Integral with respect to time of the along-track component of the wind forecast entered into the FMS descent winds page

$N \quad$ Number of observations

$p \quad$ Number of terms in regression model, including the constant

r Least squares residual

$S_{0} \quad$ Path distance of TOD location if winds were zero

$S_{\text {TOD }}$ Path distance relative to the meter fix of TOD location

$V_{h} \quad$ Vertical speed

$W_{v} \quad$ Along-track component of wind, positive for a tailwind

$\mathbf{x}=\left(x_{0}, x_{1}, \ldots, x_{p-1}\right)^{T}$ Predictive factors in regression model

$\mathbf{Z} \quad$ Observed values of $S_{\text {TOD }}$

Symbols

$\boldsymbol{\beta}=\left(\beta_{0}, \beta_{1}, \ldots, \beta_{p-1}\right)^{T}$ Coefficients of regression model

$\boldsymbol{\epsilon} \quad$ Regression model error

\section{Introduction}

$\mathrm{T}^{\mathrm{N}}$ congested airspace today, controllers direct aircraft to descend in steps. Since air density, and hence $I_{\mathrm{drag}}$, increase as the aircraft descends, significant reductions in fuel consumption and emissions would result if aircraft stayed at cruise altitude longer and then descended smoothly at idle thrust. The flight

*Aerospace Engineer, Flight Trajectory Dynamics and Controls Branch, Mail Stop 210-10, AIAA Senior Member. 
management system (FMS) on a large jet can compute the location of top of descent (TOD) assuming an idle-thrust descent. To merge aircraft, however, controllers impose level flight segments, which make it much easier for them to estimate the relative speeds of two aircraft given their calibrated airspeed (CAS). Three-Dimensional Path Arrival Management (3DPAM) enables arriving aircraft to fly optimized descents computed by the FMS in congested airspace. ${ }^{1}$ The 3DPAM ground automation must accurately predict descent trajectories in order to minimize conflicts and to provide situation awareness to controllers. To support development of the predictor and its uncertainty models, commercial flights executed idle-thrust descents at a specified descent speed, and the recorded data included not only the radar data but also the specified descent speed profile, aircraft weight, and the winds entered into the FMS descent wind page. The procedures and data collected will be explained in more detail in Section II.

Due to the potential fuel savings and emissions reductions, enabling continuous descents is being pursued by several research groups. ${ }^{2-6}$ Most of the previous error analysis has focused on the difference between operational arrival time at a waypoint and its prediction by the FMS or ground automation. In addition to predicting the meter fix arrival time accurately to ensure lateral separation, accurate prediction of the vertical profile is essential to ensure vertical separation from aircraft at different altitudes, including crossing traffic, so that controllers do not have to interrupt an FMS-controlled efficient descent. Using data from commercial operations, the current study investigated the extent to which various factors determine the TOD location and analyzed the relative importance of those factors.

Various authors ${ }^{2,5,7}$ have used operational commercial data to analyze the accuracy of the TOD location predictions from a proposed ground automation system, but such analyses do not give insight into the inherent predictability of the TOD location. Furthermore, they did not investigate the relative importance of input factors. On the other hand, analyses of the equations of motion, ${ }^{8}$ performance models, ${ }^{9}$ and FMS test benches ${ }^{6,10,11}$ have been used to assess the sensitivity of trajectories to various factors, but operational data are required to determine the sensitivity and uncertainty that an operational concept such as 3DPAM will need to handle. Green, Grace, and Williams ${ }^{12}$ did discuss the relative impact of various factors on the error in Efficient Descent Advisor (EDA) descent predictions, but they used a single research aircraft whose FMS trajectory predictor was developed by NASA rather than being a commercial design.

The primary goals of the research in this paper were to investigate the factors affecting TOD location in idle-thrust descents planned and flown by commercial FMSs and also to estimate the randomness in these TOD locations. Some factors that might affect these descents - such as engine type, winglets, FMS code revision - were not recorded during this experiment, so their effects would actually appear as noise in the trajectories. Other possible sources of noise include execution randomness or error, manually recording data incorrectly, and pilot errors entering data into the FMS. Determining whether the actual trajectory can be accurately predicted under operational conditions requires data that contain noise that would occur in operations. To account for this noise, the current research used a statistical multiple linear regression approach on the TOD locations from a relatively large sample - about 70 descents for each of two aircraft types - as discussed in Section III. Section III.E discusses the relative importance of the predictive factors, which has implications for developing an acceptable ground automation trajectory predictor for 3DPAM.

\section{Experiment Procedures and Data}

This section describes the experiment procedures and data. Coppenbarger et al. ${ }^{13}$ give more information about the procedures. In the 3DPAM concept, the FMS will be given the descent speed profile as well as the initial and final speeds and altitudes. Assuming idle thrust in the descent, the FMS will compute the vertical profile and fly it with minimal human intervention. To collect data from commercial aircraft operations, the concept was adapted to current FAA and airline procedures. The data collection occurred September 8-23, 2009, in the Denver (ZDV) Air Route Traffic Control Center (ARTCC). Two airlines participated. Eligible flights were Airbus 319/320 and Boeing 737-300, 737-800, and 757-200 descending to Denver International Airport (DEN). For simplicity in this document, the Airbus 319/320 category will simply be referred to as Airbus, and the Boeing 757-200 will be referred to as B757.

In addition to the usual speed and altitude constraints at the meter fix, the controller specified the speed profile, which was recorded by subject matter experts at ZDV. The pilot programmed the FMS to fly an idle-thrust descent, using this speed profile and the wind forecast provided by the airline. To compute its descent trajectory, the FMS used the constraints shown in Figure 1, which uniquely determine the trajectory. The parameters shown in each box are specified for that segment or that point of the descent, and their 
values - other than idle thrust - were either recorded during the experiment or extracted from radar data afterwards. The cruise speed was maintained at the initial Mach number. The initial plan was to use descent speeds 260,280 , and $300 \mathrm{kt} \mathrm{CAS} \mathrm{(KCAS)} \mathrm{roughly} \mathrm{evenly;} \mathrm{but} 260 \mathrm{KCAS}$ was only used by about $15 \%$ of the flights because it is quite slow and seemed to be penalizing crews participating in the experiment. The FMS also used speed and altitude constraints at the meter fix, which were typically to cross at 19,000 ft (16,000 ft or $17,000 \mathrm{ft}$ in $15 \%$ of descents) and at $250 \mathrm{KCAS}$ (230 KCAS in less than $5 \%$ of descents). In Figure 1, the first segment after TOD has constant Mach number, which is the same as the cruise Mach number. As the altitude decreases, the CAS increases until it reaches the target descent CAS, which was specified by the controller. The next segment is then flown at that CAS. Finally, the aircraft pitched up to decelerate to the meter fix speed constraint. In about $10 \%$ of the descents analyzed, the descent CAS was slower than the cruise speed; so the aircraft decelerated at the cruise altitude, and there was then no constant Mach segment after TOD. Once the clearance was given, the controllers and pilots then endeavored to allow the FMS to fly the descent to the meter fix with minimal human intervention. When the aircraft entered the Terminal Radar Approach Control Facility (TRACON), pilots and controllers reverted to today's standard operating procedures.

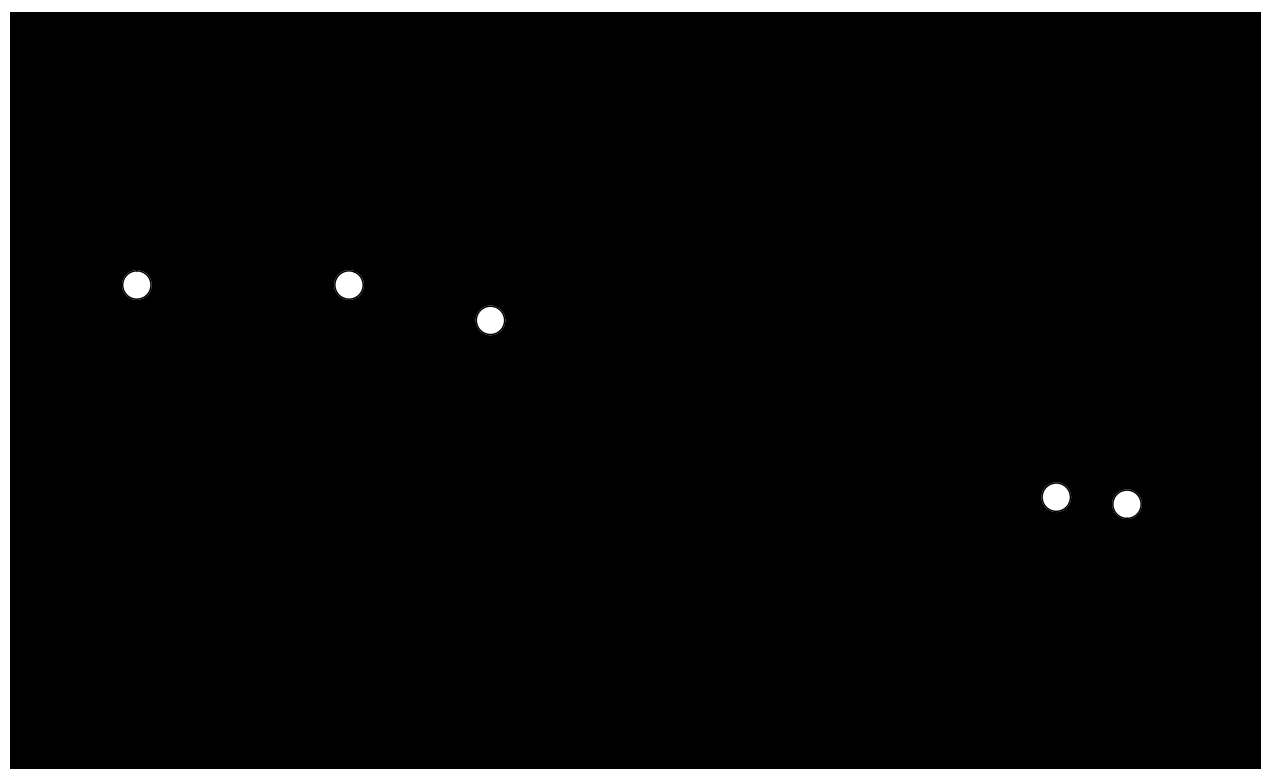

Figure 1. Schematic of typical idle-thrust descent vertical profile and constraints.

During the data collection, the Host radar data were recorded at NASA from both the ARTCC and the TRACON. The latter source was included because it extends beyond the TRACON boundary and is sent at a higher frequency than the ARTCC radar data. Due to missing data caused by a short network connectivity problem, ATAC Corporation provided Performance Data Analysis and Reporting System (PDARS) data from the same source for eight participating flights. For analysis, the horizontal flight path, cruise and meter fix altitudes, and actual TOD location were extracted from the radar data. Pilots also were requested to complete data forms that included aircraft weight, the winds programmed into the FMS, and any comments; but many pilots did not complete their data forms.

The crews of over 300 flights requested to participate. The controllers denied some requests or had to break off the procedure due to traffic or other conditions. In some cases, the radar data or pilot data sheet indicated an issue such as descent clearance issued too late, change of clearance during descent, or different speed profiles recorded on flight deck and at ZDV. In the Airbus, the flight crew must manually initiate the descent. In the B757, the autopilot system will automatically start the descent near the FMS-computed TOD, but the flight crew can of course override this and start descent either earlier or later than intended by the FMS. For this data collection, the pilots were instructed to use autopilot to the extent possible and, in the Airbus, to initiate descent near the FMS-computed TOD. Some of the sample flights, however, clearly descended more than $5 \mathrm{nmi}$ earlier than the FMS intended. In such cases, the autopilot maintains a shallow descent rate of $1000 \mathrm{ft} / \mathrm{min}$ until the aircraft intersects the intended vertical profile, at which point 
the autopilot resumes flying the FMS intent. The initial descent at $1000 \mathrm{ft} / \mathrm{min}$ was fairly easy to spot by plotting the vertical profile. Some of the flights that descended too early were identified during initial data cleansing, whilst others were discovered because they were residual outliers in multiple regression. In this paper, no such descents are included in any of the analyses. Of the roughly 20 flights discarded for descending too early, most are in Airbus aircraft and most did not have a pilot data sheet filled out. While checking whether a flight descended early was fairly easy, the only way to confirm that an aircraft descended late was from comments on the pilot data sheet or from comments recorded by the observer at ZDV. About 10 flights were discarded for such concerns, but one descent was kept due to lack of such evidence even though its vertical profile looks like it descended late. Discarding all descents with any data problems left about 110 descents each for Airbus and B757.

The aircraft weight and the winds programmed into the FMS were essential for the multiple regression analysis discussed below. Airbus and B757 each had about 70 good descents with all the necessary data. The number of B737 descents was much smaller and had to be broken into two subtypes - B737-300 and B737-800 - each of which had 10 good descents with all necessary data. This was not enough for this analysis, so the rest of this paper considers only the Airbus and B757 descents.

To make analysis of the TOD location as simple as possible, it was specified as path distance relative to the meter fix. Due to noise in the radar positions, path distance could not be computed by summing the distances between consecutive points. Instead, a piecewise linear horizontal trajectory was deduced from the radar data, either algorithmically or manually. Figure 2 shows an example radar track and estimated horizontal trajectory. The deduced horizontal trajectory ends at the meter fix and is $120 \mathrm{nmi}$ long. The cross-

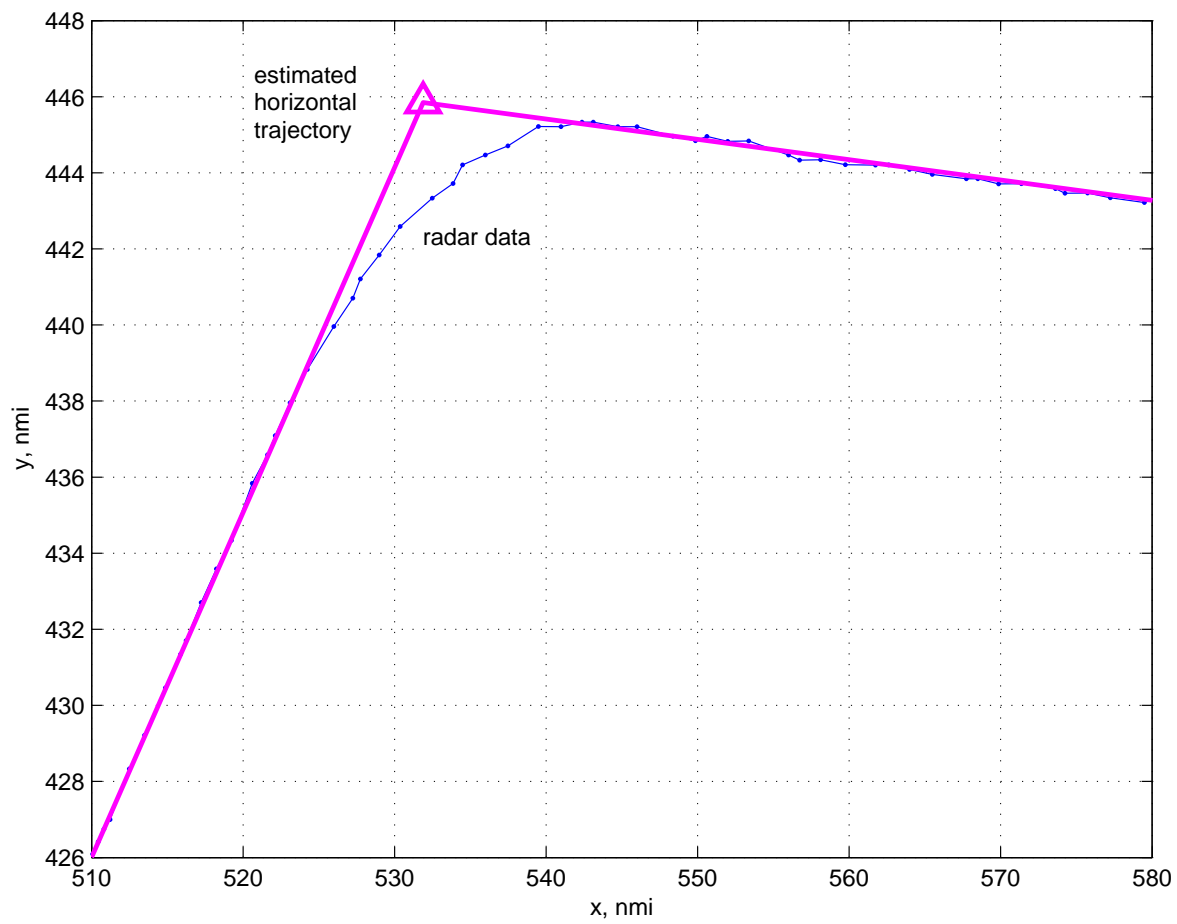

Figure 2. Example radar track and piecewise-linear estimate of the horizontal trajectory deduced from it.

track error between the radar positions and the estimated horizontal trajectory was nearly always less than $0.5 \mathrm{nmi}$, although this error was a bit larger within $10 \mathrm{nmi}$ of the meter fix because the TRACON controllers sometimes started vectoring the aircraft before it had quite reached the TRACON. For five descents, this error exceeded $5 \mathrm{nmi}$ for a few radar hits. Since the horizontal trajectory used to compute path distance assumes a zero turn radius, the TOD path distance may not be exactly right, but the FMS most likely made the same assumption. Only about $20 \%$ of the descents used in the regression analysis had any turns in the estimated horizontal trajectory. The regression residual did not seem to be affected by these turns, but identifying such an effect would be difficult since so few descents had turns. 
To complete the description of the data extracted from the radar data, the TOD location was chosen to be the point where the aircraft left cruise altitude. About 50 of the participating flights were assigned temporary altitudes within $120 \mathrm{nmi}$ of the meter fix, so the temporary altitude was used as the cruise altitude. Unfortunately, the existence of temporary altitudes as well as noise in the radar altitudes complicated determination of the cruise altitude. Essentially, the algorithm to identify the cruise altitude looked for nine consecutive radar reports with altitude within $250 \mathrm{ft}$ of each other. A couple of descents were not used because there were too few radar reports in the temporary altitude segment.

\section{Multiple Regression of Actual TOD Location}

The TOD location was modeled as a function of the following predictive factors: aircraft type, cruise altitude, cruise Mach number, descent CAS, meter fix altitude constraint, meter fix speed constraint, aircraft weight, and wind. There may be other factors that affect TOD location, but they were not recorded in these data and are not likely to be available to 3DPAM in an operational environment. The first goals of the study were to:

- determine the shape of this function, which will show the relative importance of these factors, and

- estimate the amount of noise in TOD location that is not explained by these factors, which will indicate how difficult it is to predict the TOD location.

Specifying the TOD location as path distance $S_{\text {TOD }}$ along the estimated horizontal trajectory relative to the meter fix makes the dependent variable a scalar, and all the potential independent variables except aircraft type and wind are scalars. Different aircraft types were analyzed separately. Wind was transformed to a scalar as described in Section III.A. Hence, the goal was to model a continuous scalar $S_{\text {TOD }}$ as a function of scalar variables based on empirical data. It is difficult to visualize $S_{\text {TOD }}$ as a function of all seven independent variables simultaneously. In a controlled environment, the test matrix can be chosen so that $S_{\text {TOD }}$ can be plotted as a function of each independent variable separately as in Ref. 11. Since this was an operational environment, however, the descent CAS was the only variable that could be controlled experimentally. Furthermore, the measured values of the variables included random error due to a wide variety of error sources such as radar measurements, manually recording data, deducing the horizontal trajectory and $S_{\mathrm{TOD}}$, and execution error (especially in manually initiating the descent in the Airbus).

Since $S_{\mathrm{TOD}}$ is expected to be a smooth function of the independent variables, it can be approximated by a Taylor polynomial around a point in the range of observed values of the predictive factors. Following common practice, we started with a first-order polynomial, which turned out to be sufficient. This meant the model has the form

$$
S_{\mathrm{TOD}} \approx \beta_{0}+\sum_{i} \beta_{i} x_{i},
$$

where the $x_{i}$ were chosen from the available predictive factors listed above. In matrix form, the model is

$$
\mathbf{Z}=X \boldsymbol{\beta}+\boldsymbol{\epsilon}
$$

where $\mathbf{Z}$ is an $N \times 1$ matrix containing the observed values of $S_{\text {TOD }}$; each row of the $N \times p$ matrix $X$ contains the observed values of the independent variables for one sample descent, including a one in the first column; $\boldsymbol{\epsilon}$ is the model error; $N$ is the number of observations; and $p$ is the number of coefficients. The goal was then to estimate the coefficients $\boldsymbol{\beta}$ and the error $\boldsymbol{\epsilon}$.

Since the system $\mathbf{Z}=X \boldsymbol{\beta}$ is overdetermined and has no exact solution, multiple regression uses the least squares solution

$$
\mathbf{Z}=X \mathbf{b}+\mathbf{r} .
$$

The vector $\mathbf{b}$ is an estimate of $\boldsymbol{\beta}$ that minimizes the square $\mathbf{r}^{T} \mathbf{r}$ of the residual, which is an estimate of the error $\boldsymbol{\epsilon}$. Although the use of Eq. (1) was motivated by Taylor polynomials, the regression approximation $X \mathbf{b}$ is generally not the same as the first-order Taylor polynomial. If the regression approximation is good, however, then it will be close to the Taylor polynomial. Furthermore, the goal was simply to determine a good linear approximation of $S_{\mathrm{TOD}}$ as a function of $\mathbf{x}$, if possible. 


\section{III.A. Integral of Along-track Wind}

To motivate the definition of the scalar representing wind, consider two trajectories for which all the parameters are the same except one has zero wind and the other has along-track wind $W_{v}$, with $W_{v}$ positive for a tailwind. Suppose that the total derivative $d W_{v} / d t$ along the trajectory is negligible, which is assumed by at least some FMSs. Then $W_{v}$ does not appear explicitly in the equations of motion, so the flight path angle relative to the air mass is the same function of altitude for the two trajectories. Let $S_{0}$ denote the TOD path distance of the one with zero wind and $S_{\mathrm{TOD}}$ that of the other. Then

$$
S_{0}-S_{\mathrm{TOD}} \approx \int_{t_{\mathrm{TOD}}}^{t_{\mathrm{fix}}} W_{v}(\tau) d \tau=\int_{h_{\mathrm{TOD}}}^{h_{\mathrm{fix}}} \frac{W_{v}(h)}{V_{h}(h)} d h,
$$

where $t$ is time, $h$ is altitude, and $V_{h}$ is the vertical speed of the trajectory with nonzero wind. This is true because the integral is approximately the additional distance that the wind moves the aircraft with respect to the ground between TOD and the meter fix. This means the term in Eq. (1) due to wind should be

approximately $-\int_{t_{\mathrm{TOD}}}^{t_{\mathrm{fix}}} W_{v}(\tau) d \tau \equiv-I_{\mathrm{W}, \mathrm{FMS}}$, and this would be exactly true if the derivative of wind were omitted from the equations of motion when computing $S_{\mathrm{TOD}}$.

The formula to compute $I_{\mathrm{W}, \mathrm{FMS}}$ for this analysis was based on integrating with respect to altitude because wind was input to the FMS as a function of altitude only. We then assumed that the $x$ and $y$ coordinates of wind were linear between the altitudes input to the FMS (which is probably not how the FMS interpolates wind), and approximated $V_{h}$ by the constant $-2000 \mathrm{ft} / \mathrm{min}$ in order to avoid estimating a derivative from noisy data. The value of $I_{\mathrm{W}, \mathrm{FMS}}$ was then computed using the trapezoidal rule with nodes at the waypoints on the piecewise linear horizontal trajectory and at the altitudes at which the wind conditions were specified to the FMS (typically cruise altitude, FL340, FL300, FL240, and FL180).

\section{III.B. Selecting Regressors}

The objective in this section is to determine which of the candidate predictive factors actually affect $S_{\text {TOD }}$. This was simplified by the fact that the factors were independent of each other, and the order in which they were added to the model did not matter. First, meter fix speed was discarded as a possible predictive factor because very few (less than 5 per aircraft type) of the descents had a speed constraint other than $250 \mathrm{KCAS}$ at the meter fix. The next question was whether to use all six remaining predictive factors. The fit always improves as more factors are added to the model, but overfitting should be avoided. There are several methods to select the regressors: partial- $F$ tests (MATLAB Statistics Toolbox stepwisefit function with default settings), using confidence intervals or $p$-values for the estimated coefficients, comparing residuals, and comparing $R^{2}$ values. In this case, all gave the same results for all six candidate factors.

For the Airbus, the model should include the cruise and meter fix altitudes, descent CAS, wind, and aircraft weight. The $R^{2}$ value was then 0.93 , and adding cruise Mach only increased it by 0.002 . Figure 3 shows the distributions of the residuals for selected sets of regressors, which confirms that adding cruise Mach has little effect on the residuals for the Airbus. Using the empirical cumulative distribution function rather than histograms in this figure makes it much easier to compare four distributions in the same plot. It also makes it easy to estimate the percentage of descents with residuals in any specified range. Figure 4 also shows the histogram of the residuals for the models with the selected regressors.

In the plot of residuals for the B757, however, adding weight had very little effect, so the red line essentially covers the green line. Including the cruise and meter fix altitudes, descent CAS, and wind gave an $R^{2}$ value of 0.92 , and adding aircraft weight and cruise Mach only increased it by 0.0002 .

This does not necessarily mean that these values are not used by the FMS or that they have no effect on the FMS-computed trajectory. Instead, it means that their effects were too small to be observed in this data set, given the noise and the observed ranges of the parameters. Assuming Standard Atmosphere to convert the cruise Mach numbers to true airspeed showed that, for each aircraft type, a spread of about $15 \mathrm{kts}$ covered about $65 \%$ of the descents used in this regression. Thus, the observed variation in cruise Mach was not large enough to cause much variation in TOD location. The difference between the minimum and maximum weights was about the same for the two aircraft types, but apparently the partial derivative of $S_{\mathrm{TOD}}$ with respect to weight was larger for the Airbus than for the B757.

Note that Figure 4 shows that all but six of the observations have residual with absolute value less than $5 \mathrm{nmi}$, which is a tentative bound on acceptable error for the ground automation trajectory predictor. Thus, these predictive factors adequately explain the TOD location, which means the data do not include excessive 


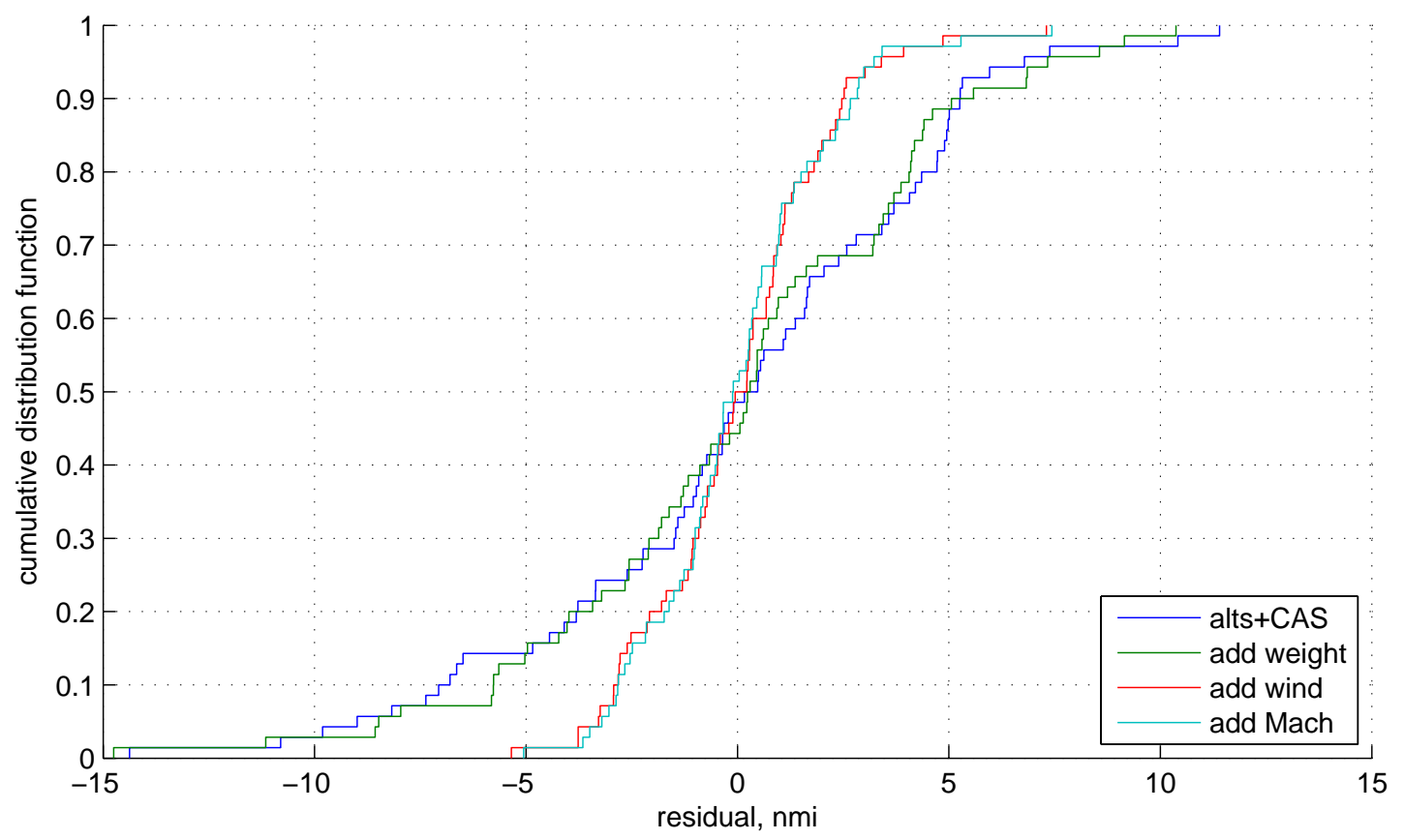

(a) Airbus

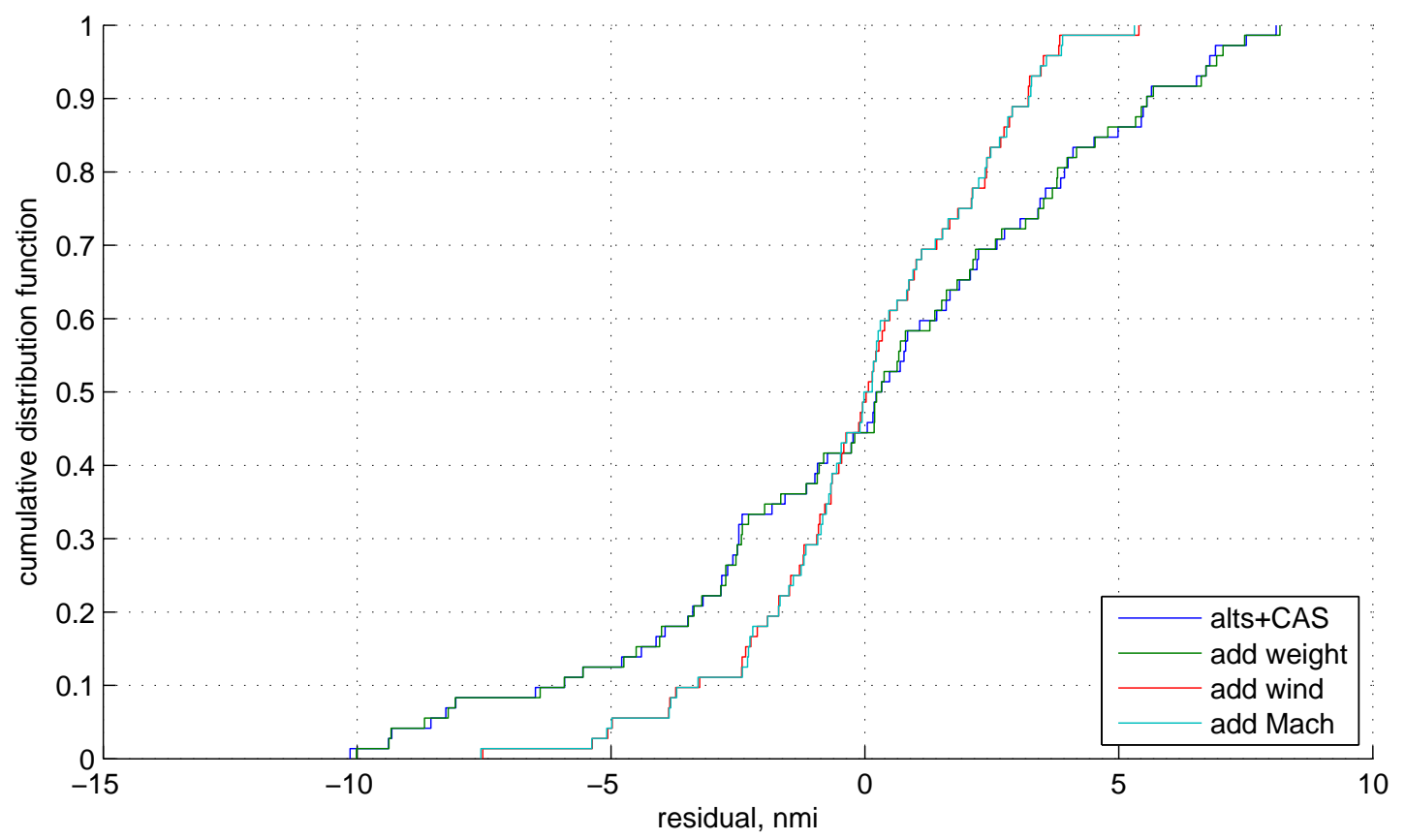

(b) B757

Figure 3. Cumulative distribution functions of residuals for regression of actual TOD path distance for selected sets of regressors. 


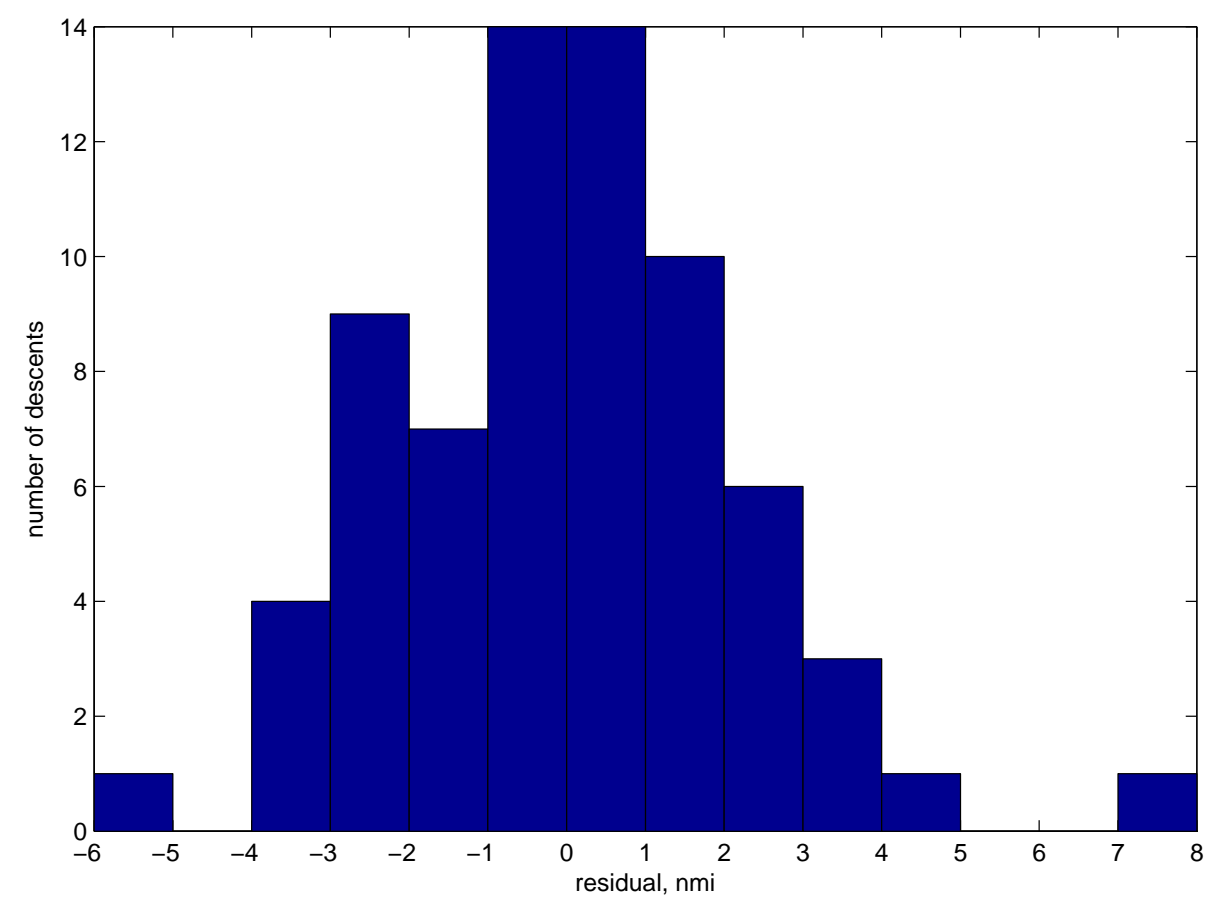

(a) Airbus

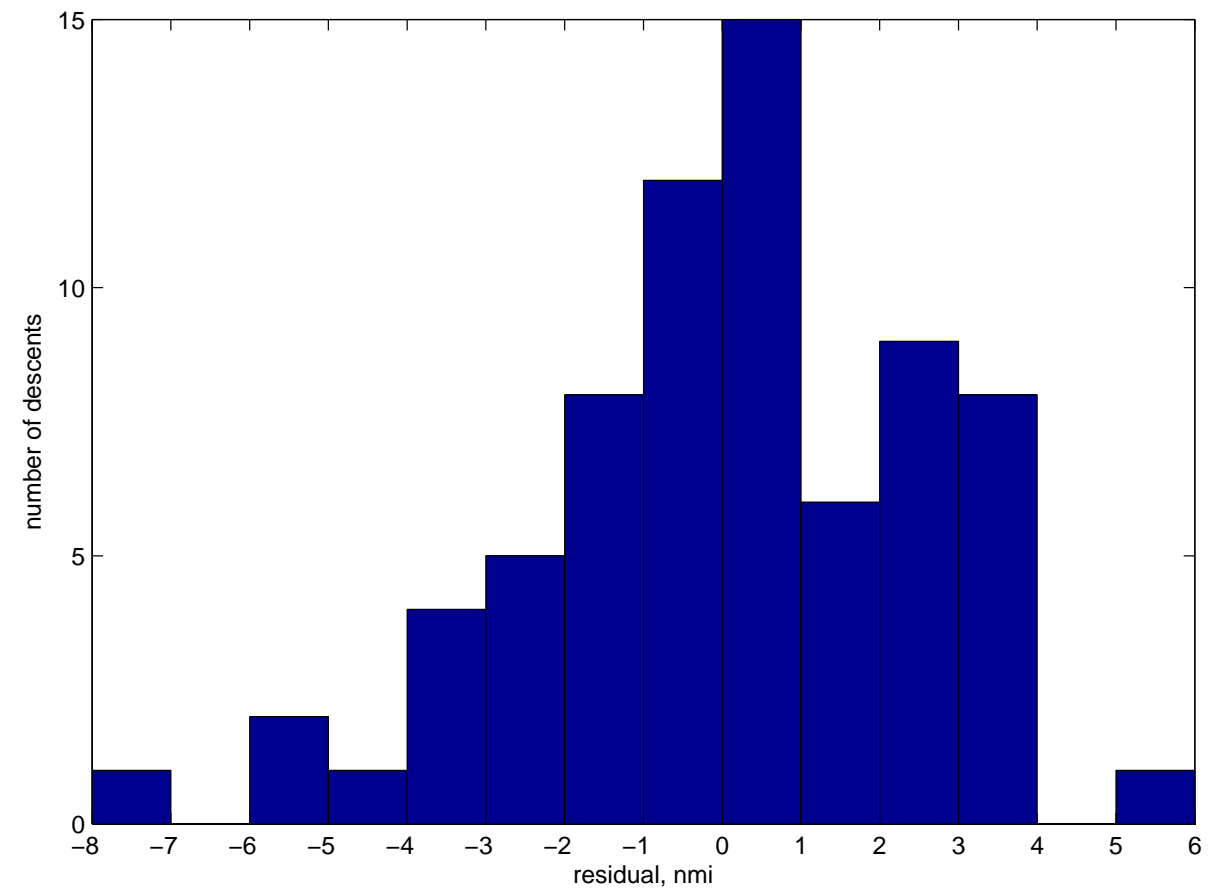

(b) B757

Figure 4. Histograms of regression residuals for (a) Airbus when the model includes cruise and meter fix altitudes, descent CAS, wind, and aircraft weight and (b) B757 when the model includes the same regressors except weight.

\section{8 of 12}


noise. Furthermore, any additional factor affecting TOD location either has a small effect or changes it by an additive constant for these descents. The approximate linearity is consistent with Stell, ${ }^{11}$ which only investigated the effect of descent speed, aircraft weight, and wind.

\section{III.C. Estimating Coefficients}

This section gives the estimated coefficients of the regression model. If the regression assumptions are valid, then regression not only gives estimates $b_{i}$ of the coefficients in the model but also confidence intervals for $\beta_{i}$. If the experiment were run 100 times, 95 of the resulting 95\%-confidence intervals for $\beta_{i}$ would include the true value of $\beta_{i}$. If the $95 \%$-confidence interval for $\beta_{i}$ does not include zero, then the null hypothesis that $\beta_{i}$ is equal to zero would be rejected at the $95 \%$ level; so this gives another way to assess which candidate predictive factors are significant. Including all the possible factors in the model resulted in $95 \%$-confidence levels that included zero for the coefficient of cruise Mach number for both aircraft types, and also the interval for the coefficient of weight includes zero for the B757. Including only the significant terms in the model for each aircraft type gave the estimates and 95\%-confidence intervals for the coefficients shown in Table 1.

Table 1. Estimates and confidence intervals of the coefficients from multiple regression of actual TOD path distances.

\begin{tabular}{|c|c|c|c|c|}
\hline \multirow[b]{2}{*}{ Term } & \multicolumn{2}{|r|}{ Airbus } & \multicolumn{2}{|r|}{$\mathrm{B} 757$} \\
\hline & $b_{i}$ & $\begin{array}{c}95 \% \text {-confidence } \\
\text { interval for } \beta_{i} \\
\end{array}$ & $b_{i}$ & $\begin{array}{c}95 \% \text {-confidence } \\
\text { interval for } \beta_{i} \\
\end{array}$ \\
\hline Constant & -14 & {$[-41,12]$} & -76 & {$[-97,-54]$} \\
\hline Cruise altitude, $\mathrm{ft}$ & -0.0028 & {$[-0.0030,-0.0025]$} & -0.0025 & {$[-0.0028,-0.0023]$} \\
\hline Meter fix altitude, $\mathrm{ft}$ & 0.0027 & {$[0.0018,0.0035]$} & 0.0027 & {$[0.0021,0.0033]$} \\
\hline Descent CAS, kt & 0.14 & {$[0.095,0.18]$} & 0.20 & {$[0.15,0.25]$} \\
\hline Wind integral, nmi & -1.3 & {$[-1.5,-1.2]$} & -1.1 & {$[-1.3,-0.95]$} \\
\hline Weight, lb & -0.00027 & {$[-0.00035,-0.00019]$} & omit & d from model \\
\hline
\end{tabular}

For the B757, Table 1 shows the results for a regression model that excludes aircraft weight because it does not seem to be significant. Including weight would increase the constant term by $4 \mathrm{nmi}$ but would not affect the other coefficients at the precision shown. The estimate of the coefficient of weight would be on the order of $-10^{-5}$ with the endpoints of the $95 \%$-confidence interval being $\pm 10^{-4}$.

The coefficient of the integral $I_{\mathrm{W}, \mathrm{FMS}}$ of the along-track component of wind merits a few comments. Section III.A indicated that this coefficient should be -1 . This is close to its estimate for the B757 but is outside the 95\%-confidence interval for the Airbus estimate. There are two likely causes of this discrepancy. One is that the FMS uses the current wind measurement blended with the forecast conditions entered by the pilot in computing the optimal TOD location, whereas $I_{\mathrm{W}, \mathrm{FMS}}$ does not involve current measurements. Another is that the value of $I_{\mathrm{W}, \mathrm{FMS}}$ was computed assuming a constant value for $V_{h}$. The preceding results indicate, however, that simply using $I_{\mathrm{W}, \mathrm{FMS}}$ in the regression gave a model that fits the data reasonably well.

\section{III.D. Regression Assumptions and Caveats}

Multiple regression is based on several assumptions on the model error $\boldsymbol{\epsilon}$. While the least squares solution of Eq. (3) does not depend upon these assumptions, the statistical techniques associated with regression do depend upon them. For example, the partial- $F$ tests and coefficient $p$-values mentioned in Section III.B for selecting regressors depend upon these assumptions. Ultimately, however, the selection of regressors was based on distributions of residuals shown in Figure 3, which is based only on the least squares solution. More importantly, the regression assumptions are essential for the confidence intervals in Table 1. This section briefly considers the validity of these assumptions.

One of the assumptions is that the error is normal. This is typically checked visually with a normal probability plot, but this is a qualitative rather than quantitative test. Another approach is to use a statistical test to compare the empirical distribution to the normal distribution. In this case, the $\chi^{2}, z, t$, 
and Kolmogorov-Smirnov tests did not reject the assumption (all $p$-values greater than 0.3 for both aircraft types). The most useful approach in this analysis, however, was to check the residual outliers, which were those residuals with absolute value greater than twice the standard deviation of all the residuals. This helped identify several of the aircraft that descended early as mentioned in Section II. A further test was to check for influential observations, which are those that significantly change the regression results when they are added to the other points. Using standard techniques (Cook's distance with threshold 1 and DFFITS ${ }^{14}$ ) to identify any such observations and leaving them out of the regression did not change the estimated coefficients by much more than $10 \%$ except for the constant terms and descent CAS coefficients. For both aircraft types, the new coefficient of descent CAS was 0.17. Therefore, influential observations did not seem to be a problem.

The model errors should also be independent. Plotting the residuals versus descent times suggests the error distribution changes over time. This may be due to the fact that the FMS merged the current wind measurement with the wind forecast and forecast error changes with time, but it will be investigated in future research. Furthermore, when interaction and quadratic terms are included in the possible regressors, the final model determined by the MATLAB stepwisefit function with default settings (except for scaling) replaced some of the linear terms included previously with products of those terms. This suggests multicollinearity might be a problem, which could affect the results in Section III.E, and will be investigated more in the future.

Using random regressors also might cause problems, but the goal here was to model TOD path distance as a function of the stated or recorded values of the regressors, so the errors in these values did not affect the regression itself. More importantly, the observed values of the predictive factors do not adequately cover their likely ranges in an operational system. Almost $90 \%$ of the regression samples have an altitude constraint of $19,000 \mathrm{ft}$. All but $15 \%$ of the samples have descent speed either 280 or 300 KCAS. The operational spread of $I_{\mathrm{W}, \mathrm{FMS}}$ is unknown. This shortcoming must be addressed in future data collection.

Finally, the discarding of the early and late descents as well as analyzing only those descents with completed pilot data forms raise questions about how well the errors represent real-world errors. This is known as censoring data. If 3DPAM were deployed, pilots would be thoroughly trained on the procedure and would be expected to follow it. Pilot error could still occur and the system would need to be robust to such error, but the discarded descents from this data collection probably do not represent the error in an operational system.

\section{III.E. Relative Importance of Predictive Factors}

The regression model of $S_{\text {TOD }}$ helps to understand the general problem of predicting TOD location for these descents. This section estimates a lower bound on error due to using a nominal value for a predictive factor. This also indicates the relative importance of the predictive factors.

Since typical values of descent CAS and cruise altitude, say, differ by several orders of magnitude, simply comparing the magnitudes of their coefficients does not indicate their relative importance in predicting $S_{\text {TOD }}$. Suppose that one were developing a trajectory predictor that used a nominal value $v_{0}$ for descent speed instead of the actual value $v$. Suppose also that there were no other sources of error. The regression results show that $S_{\mathrm{TOD}}$ as a function of $v$ can be approximated by a line as shown in Figure 5 . The slope of the line would be $b_{v}$, where $b_{v}$ is the regression coefficient of the descent speed, so the error in $S_{\mathrm{TOD}}$ due to using $v_{0}$ instead of $v$ would be $\Delta S_{\mathrm{TOD}}=b_{v}\left(v-v_{0}\right)$. To minimize this error, choose $v_{0}$ to be the midpoint of the range $\left[v_{\min }, v_{\max }\right]$ of descent speeds. Hence, the error bound on $S_{\mathrm{TOD}}$ would be

$$
\left|\Delta S_{\mathrm{TOD}}\right| \leq \frac{1}{2}\left|b_{v}\right|\left(v_{\max }-v_{\min }\right) .
$$

The same argument would obviously apply to any of the other predictive factors. Let factor $x_{i}$ have bounds $\left[x_{i, \min }, x_{i, \max }\right]$, and define

$$
\left(\Delta S_{\mathrm{TOD}}\right)_{i}=\frac{1}{2}\left|b_{i}\right|\left(x_{i, \max }-x_{i, \min }\right) .
$$

Comparing the $\left(\Delta S_{\mathrm{TOD}}\right)_{i}$ values to each other also shows the relative importance of the factors in determining $S_{\text {TOD }}$. Table 2 shows estimates of the minimal ranges of values likely to be encountered by an operational system along with the corresponding error bound $\left(\Delta S_{\mathrm{TOD}}\right)_{i}$. The rows in the table are in order of decreasing $\left(\Delta S_{\mathrm{TOD}}\right)_{i}$, which means they are also in order of decreasing importance. This table omits B757 weight due to the relatively large uncertainty in its estimated coefficient. Furthermore, the coefficients of the two altitudes 


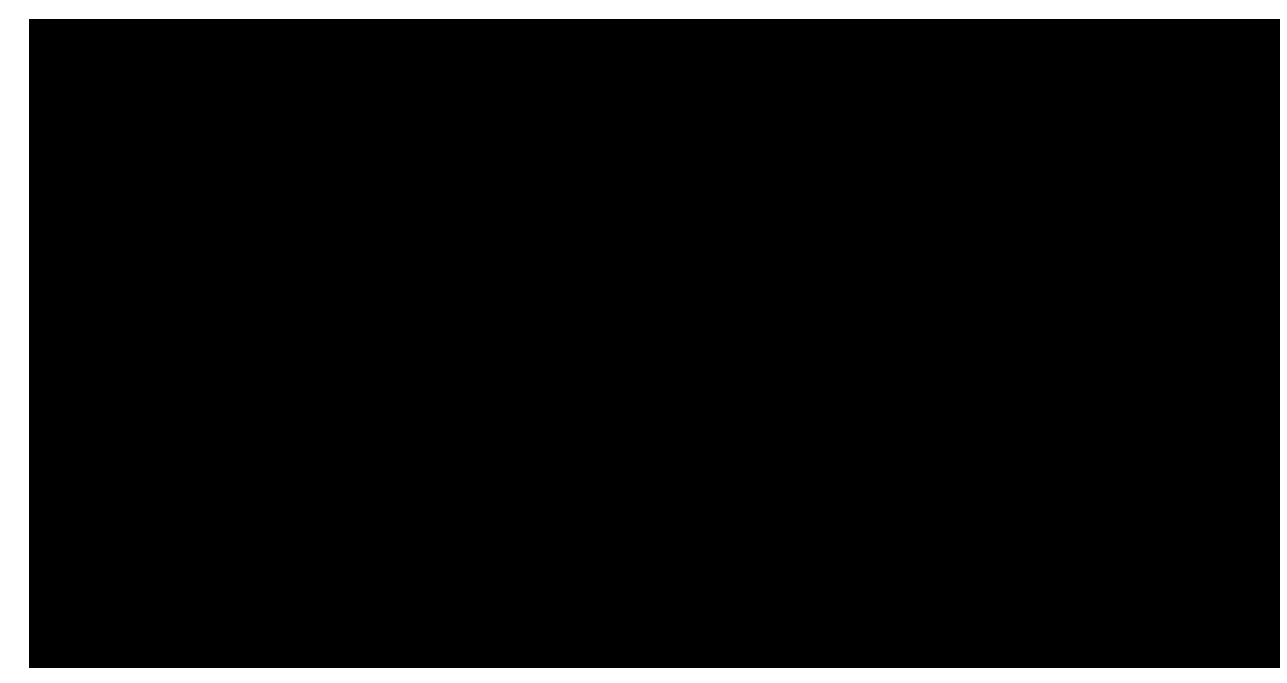

Figure 5. Depiction of $\Delta S_{\text {TOD }}$.

in Table 1 are roughly the negative of each other, which means the cruise and meter fix altitudes can be replaced in this table by the change in altitude between TOD and the meter fix, with estimated coefficient -0.0027 for both aircraft types.

Table 2. Approximate lower bound on TOD location prediction error due to a nominal value of an independent variable.

\begin{tabular}{l|c|lc}
\multirow{2}{*}{$x_{i}$} & & \multicolumn{2}{|c}{$\left(\Delta S_{\mathrm{TOD}}\right)_{i}, \mathrm{nmi}$} \\
\cline { 3 - 4 } \multicolumn{1}{c|}{$\left[x_{i, \min }, x_{i, \max }\right]$} & Airbus & $\mathrm{B} 757$ \\
\hline \hline Altitude change & {$[9000,22000] \mathrm{ft}$} & 18 & 18 \\
Wind integral & {$[-10,10] \mathrm{nmi}$} & 13 & 11 \\
Descent CAS & {$[250,320] \mathrm{KCAS}$} & 4.9 & 7.0 \\
Weight & {$[115000,143000] \mathrm{lb}$} & 3.8 & $\mathrm{~N} / \mathrm{A}$
\end{tabular}

The bounds on the predictive factors in Table 2 are primarily based on the values observed in the data, but an operational system might encounter even larger ranges. For example, the Vmo/Mmo for the Airbus 320 is $350 \mathrm{KCAS} / 0.82 \mathrm{Mach}$, so an operational system might encounter larger descent speeds than shown in Table 2 for the Airbus. The change in altitude between TOD and the meter fix will be larger at most other airports since Denver has unusually high meter fix altitude constraints.

Consequently, the values in Table 2 probably underestimate the effect on $S_{\mathrm{TOD}}$ of the predictive factors. They suggest, however, that in order to predict TOD location within $5 \mathrm{nmi}$ a predictor cannot use nominal values for altitude change, descent CAS, or wind. A TOD location predictor for the Airbus might be able to use a nominal value for aircraft weight, but keep in mind that the error budget must allow for other sources of inaccuracies.

\section{Conclusions}

The major conclusions of this paper are that:

- a predictor will need to use accurate values of cruise and meter fix altitudes, descent CAS, and wind to predict TOD location within $5 \mathrm{nmi}$ for the Airbus 320 and the B757 - and weight may also be necessary for the Airbus - and

- the observed TOD locations for these descents do not contain excessive noise, as indicated by the fact that almost all the residuals in Figure 4 have absolute value less than 5 nmi. 
Additional factors might be necessary to explain TOD location in data with more aircraft types and airlines, at more locations, over longer periods of time, with larger ranges for the predictive factors, and so forth. For example, Tong, Boyle, and Warren ${ }^{6}$ found that de-icing setting moved TOD location by about $15 \mathrm{nmi}$ on a B777 FMS test bench, but observing that in operational data would require collecting over a greater variety of meteorological conditions. For the descents analyzed here, however, failure of a trajectory predictor to compute TOD location within $5 \mathrm{nmi}$ is not due to randomness. Furthermore, if it is due to a hidden factor, it is a factor that changed TOD location by an additive constant for these descents.

The focus of this analysis was predictability of TOD location. Future work on this topic includes analyzing more data (both operational and laboratory), improving the analysis (using enhanced statistical methods and reducing assumptions such as the use of constant $V_{h}$ in computing $\left.I_{\mathrm{W}, \mathrm{FMS}}\right)$, and reproducing the regression model analytically. Many additional obstacles must be overcome in future research in order to deploy a 3DPAM system that would be effective in all traffic conditions. The ground predictor will need to have the horizontal trajectory, which was extracted from the radar data in post-operations analysis here. Furthermore, the along-track error has not been analyzed. The target TOD location error bound of $5 \mathrm{nmi}$ was chosen based on some previous controller feedback; but the tradeoffs between prediction error, conflict avoidance buffer size, and throughput should be investigated, as well as the impact of prediction error on controllers' situation awareness and workload. Most importantly, this analysis provides important insight into how to develop a ground-based trajectory predictor for a 3DPAM system and might even be the basis for such a predictor, but such a predictor still does not exist. Furthermore, even if the TOD location were known perfectly by the ground automation, the rest of the vertical profile as well as the path distance at given time must also be predicted accurately.

\section{References}

${ }^{1}$ Scharl, J., Berge, M., Coats, M. L., Haraldsdottir, A., and Schoemig, E. G., "Modeling and Analysis of the 3D Path Arrival Management Concept (AIAA 2007-6878)," AIAA Modeling and Simulation Technologies Conference, AIAA, Washington, DC, 2007.

${ }^{2}$ Coppenbarger, R. A., Mead, R. W., and Sweet, D. N., "Field Evaluation of the Tailored Arrivals Concept for Datalinkenabled Continuous Descent Approach (AIAA 2007-7778)," AIAA 5th Aviation, Technology, Integration, and Operations Conference, AIAA, Washington, DC, 2007.

${ }^{3}$ Klooster, J. K., Amo, A. D., and Manzi, P., "Controlled Time-of-Arrival Flight Trials," 8th USA/Europe Air Traffic Management RED Seminar, 2009.

${ }^{4}$ Ren, L. and Clarke, J.-P. B., "Flight-Test Evaluation of the Tool for Analysis of Separation and Throughput," Journal of Aircraft, Vol. 45, No. 1, January-February 2008, pp. 323-332.

${ }^{5}$ Roberts, C., "Australian Tailored Arrivals Trial: Phase 2A Report," Tech. rep., Boeing, Airservices Australia, Qantas Airways, Emirates, Air Traffic Alliance, July 2007.

${ }^{6}$ Tong, K., Boyle, D. A., and Warren, A. W., "Development of Continuous Descent Arrival (CDA) Procedures for Dualrunway operations at Houston Intercontinental (AIAA 2006-7750)," AIAA 6th Aviation, Technology, Integration, and Operations Conference, AIAA, Washington, DC, 2006.

${ }^{7}$ Green, S. M. and Vivona, R. A., "Field Evaluation of Descent Advisor Trajectory Prediction Accuracy (AIAA 19963764)," AIAA Guidance, Navigation and Control Conference, AIAA, Washington, DC, 1996.

${ }^{8}$ Jackson, M. R., Zhao, Y. J., and Slattery, R. A., "Sensitivity of Trajectory Prediction in Air Traffic Management," Journal of Guidance, Control, and Dynamics, Vol. 22, No. 2, March-April 1999, pp. 219-228.

${ }^{9}$ Bayraktutar, I., "Sensitivity of Trajectory Prediction to Input Data," Tech. Rep. CTP/2005/anr/ebo, EUROCONTROL, June 2005.

${ }^{10}$ Herndon, A. A., Cramer, M., Sprong, K., and Mayer, R. H., "Analysis of Advanced Flight Management Systems (FMS), Flight Management Computer (FMC) Field Observations Trials, Vertical Path," 26th Digital Avionics Systems Conference (IEEE DASC 07), IEEE, Piscataway, NJ, 2007.

${ }^{11}$ Stell, L. L., "Analysis of Flight Management System Predictions of Idle-thrust Descents," 29th Digital Avionics Systems Conference (IEEE DASC 10), IEEE, Piscataway, NJ, 2010.

${ }^{12}$ Green, S. M., Grace, M. P., and Williams, D. H., "Flight Test Results: CTAS and FMS Cruise/Descent Trajectory Prediction Accuracy," 3rd USA/Europe Air Traffic Management RछD Seminar, 2000.

${ }^{13}$ Coppenbarger, R., Dyer, G., Hayashi, M., Lanier, R., Stell, L., and Sweet, D., "Development and Testing of Automation for Efficient Arrivals in Constrained Airspace," 27th International Congress of the Aeronautical Sciences (ICAS 2010), ICAS, Stockholm, 2010.

${ }^{14}$ Ryan, T. P., Modern Regression Methods, John Wiley \& Sons, Inc., Hoboken, NJ, 2009. 